\title{
Hydrocortisone vs. dexamethasone treatment for bronchopulmonary dysplasia and their effects on general movements in preterm infants
}

\author{
Marrit M. Hitzert ${ }^{1}$, Manon J.N.L. Benders ${ }^{2}$, Annemiek M. Roescher ${ }^{1}$, Frank van Bel ${ }^{2}$, Linda S. de Vries ${ }^{2}$ and Arend F. Bos ${ }^{1}$
}

INTRODUCTION: Hydrocortisone (HC) and dexamethasone (DXM) are used to treat preterm infants at risk for bronchopulmonary dysplasia (BPD). This may, however, affect their longterm neurological development. We aimed to determine the effect of HC and DXM therapy in preterm infants on neurological functioning as assessed by the quality of general movements (GMs) until 3 months after term.

RESULTS: We found no difference in the quality of GMs between $\mathrm{HC}$ and DXM infants until term age. At 3 months, $\mathrm{HC}$ infants had a higher median motor optimality score (MOS) than DXM infants (25 vs. $21, P=0.015$ ). In the DXM group, MOS on the first day of treatment was lower than before treatment (10 vs. 11, $P=0.030$ ). DISCUSSION: MOS decreased in DXM infants on the first day following treatment and at 3 months after term. This was not the case in $\mathrm{HC}$ infants. Our study suggests that neurological functioning at 3 months after term is better in infants treated with $\mathrm{HC}$ than in infants treated with DXM.

METHODS: We performed a longitudinal, observational study including 56 preterm infants ( $n=17 \mathrm{HC}, n=17 \mathrm{DXM}, n=22$ controls). GM quality, videoed before and after treatment, was assessed. In addition, a MOS was assigned to details of the GMs.

mproved neonatal intensive care over recent decades has led to significantly increased survival of preterm infants. The incidence of bronchopulmonary dysplasia (BPD), however, is still approximately $30 \%$ (1). Previous studies investigating the effect of postnatal corticosteroid therapy showed that dexamethasone (DXM) therapy facilitates extubation and reduces the rate of BPD at 28 days after birth and at 36 weeks postmenstrual age (2). Furthermore, there is a reduction in the need for late rescue with corticosteroids (3). Unfortunately, postnatal DXM therapy is associated with short-term adverse effects and an increased risk of motor disorders, including cerebral palsy $(3,4)$, particularly if therapy is started within the first week of life (5). Various studies on postnatal hydrocortisone (HC) therapy reported an equal reduction in oxygen dependency and fewer short-term adverse effects than DXM, and no long-term adverse effects on neurological outcome as compared to controls (6,7). A twocenter comparative study reported that HC-treated children had a better cognitive and motor neurodevelopmental outcome as compared to DXM-treated children (8). In a recent systematic review, however, early postnatal HC therapy showed no improvement in mortality, the rates of BPD, or in-home oxygen dependence (9). As yet, no consensus exists regarding the most optimal therapy for preterm infants at risk for BPD.

The qualitative assessment of general movements (GMs) from video recordings is a sensitive, noninvasive method to investigate the integrity of the young infant's brain $(10,11)$. This method is based on visual Gestalt perception of GM quality up to 5 months after term. Normal GMs are characterized by complexity, variability, and fluency, whereas in abnormal GMs, these are reduced (12). The quality of the fidgety general movements (FMs), present between 9 and 20 weeks after term and defined as continuous small movements of moderate speed in all directions, is a particularly accurate marker for neurological outcome: most infants (96\%) with normal FMs have normal neurological outcomes, whereas most infants (95\%) in whom FMs are absent during this period develop cerebral palsy (13). Recent studies reported that detailed aspects of GMs, expressed as a motor optimality score (MOS), also have predictive value for mild motor abnormalities later on $(14,15)$.

Previously, DXM treatment was associated with the presence of abnormal GMs, which correlated with the severity of brain lesions and the occurrence of cerebral palsy $(16,17)$. The influence of HC therapy on short-term neurological outcome as assessed by the quality of GMs is unknown.

This led to our aim of determining the effects of HC and DXM therapy in preterm infants on neurological functioning as assessed by the quality of GMs until 3 months after term.

\section{RESULTS}

Patient Characteristics

Table 1 shows the most important patient characteristics. The HC group differed from the DXM group in that the former infants had lower birth weights, had higher Apgar scores

'Department of Pediatrics, Beatrix Children's Hospital, University Medical Center Groningen, University of Groningen, Groningen, The Netherlands; ${ }^{2}$ Department of Pediatrics, Wilhelmina Children's Hospital Utrecht, University Medical Centre Utrecht, University of Utrecht, Utrecht, The Netherlands.Correspondence: Marrit M. Hitzert

(m.m.hitzert@umcg.nl) 
at $5 \mathrm{~min}$, and received lower mean and cumulative equivalent doses of cortisol. Although not significantly different, HC infants were treated more often with sedative, anticonvulsant, or muscle-relaxant drugs during corticosteroid treatment than the DXM group (94\% and 67\%, respectively).

Brain ultrasound scans of three DXM infants deteriorated after initiating DXM treatment. In one infant, the density of the periventricular white matter increased, which was followed by a leukomalacia grade III. Two infants in whom periventricular echodensities had normalized before DXM therapy developed ventriculomegaly. All three of these infants had abnormal GMs before therapy. At 2 years of age, six of the DXM-treated children had developed cerebral palsy vs. none of the HC-treated infants $(P=0.018)$.

Data on neurodevelopmental outcome at 2 years are presented in Table 2. In each group, test results of three infants were missing either due to loss of follow-up or an insufficiently cooperating infant.

\section{Quality of GMs}

The results on the quality of GMs are shown in Figure 1. In the control group, nine infants showed normal GMs on day 0 and 13 showed abnormal GMs. We found no difference in the quality of GMs between the HC and DXM groups and the controls on day 0 . On day 7 after therapy had commenced, we found more infants in the DXM group in whom the quality of GMs was abnormal as compared to the controls. This finding just reached statistical significance $(P=0.017)$. We found differences neither between the HC group and the controls nor between the HC group and the DXM group. At 3 months after term, 18 control infants showed normal FMs and in one infant, FMs were absent. In the DXM group, the quality of FMs was abnormal in more infants as compared to the controls $(P=0.013)$. Again, we found no differences in the quality of FMs between the $\mathrm{HC}$ group and controls, and between the HC and DXM groups.

\section{MOSs in HC- and DXM-Treated Infants}

In the first week after treatment had commenced, the median MOS was higher in controls as compared to infants treated with corticosteroids (Figure 2a). This difference was significant between the DXM group and control infants (median 10 vs. 14, $P=0.010$ ) only on day 1 . We found no differences between the two treatment groups, except for MOS at 3 months after term (Figure 2b). MOS of controls (median 24) was higher as compared to the DXM group (median 21, $P=0.010$ ), and it was higher for the infants in the HC group (median 25) as compared to the infants in the DXM group $(P=0.015)$.

In addition, we analyzed the change in MOS from day 0 to day 1 , with each infant serving as his or her own control. Hypokinetic infants were given 8 points, i.e., the lowest MOS. In the HC group, two infants were hypokinetic before treatment. One of these infants showed poor-repertoire GMs on day 1 whereas for the other infant, no recording was available for day 1 . None of the infants in the DXM group was hypokinetic before corticosteroid administration, whereas two infants became hypokinetic on day 1. As shown in Figure 3, we found the median MOS

Table 1. Patient characteristics

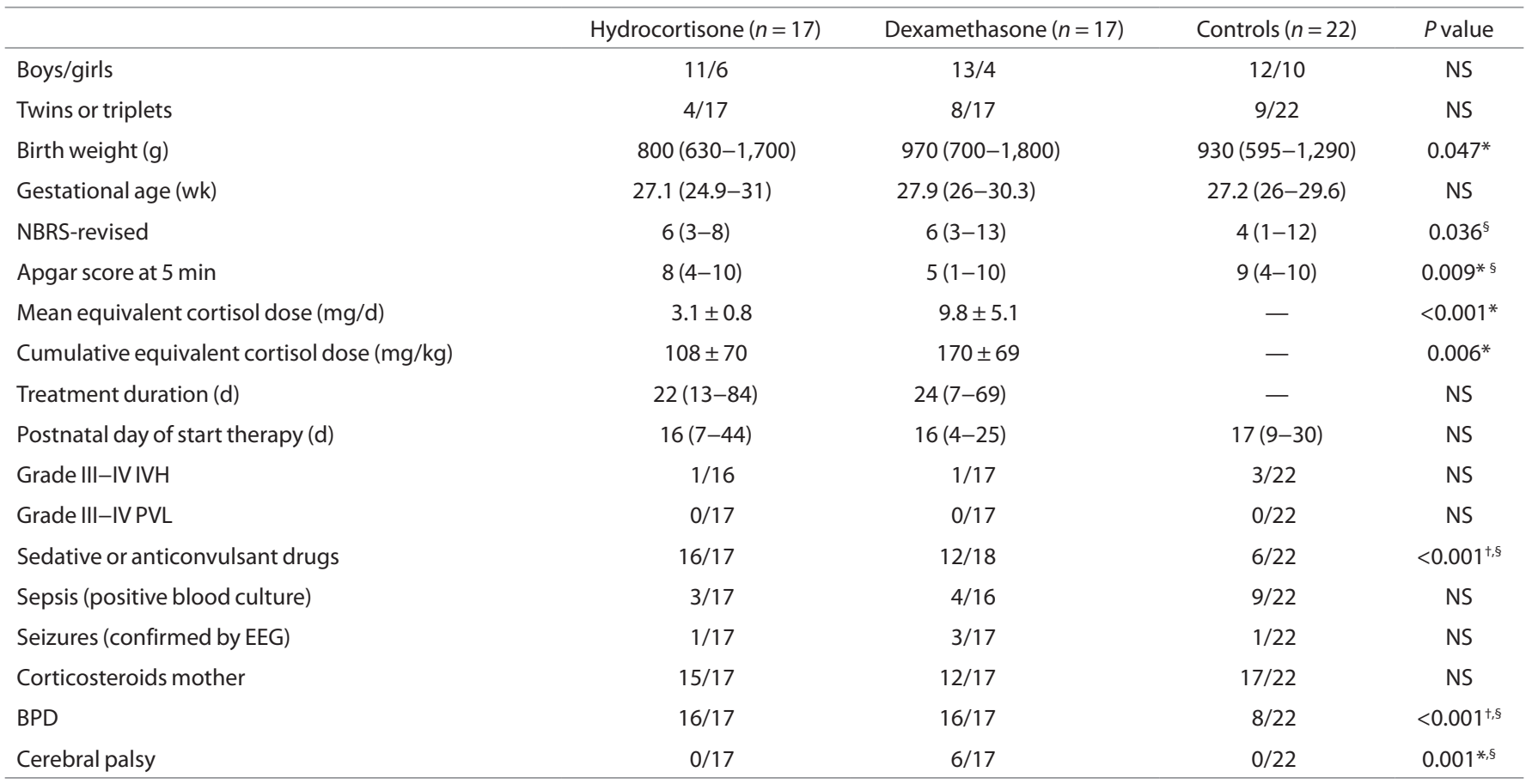

Variables represent frequencies ( $n /$ total), median (range), or mean ( \pm SD). BPD, bronchopulmonary dysplasia; EEG, electroencephalogram; IVH, intraventricular hemorrhage;

NBRS, Nursery Neurobiologic Risk Score; NS, not significant; PVL, periventricular leukomalacia. $P$ values represent statistical differences in categorical variables as calculated by the

Fisher exact test, or continuous variables as calculated by the Kruskall-Wallis test.

*Statistical differences $(P<0.1)$ between hydrocortisone and dexamethasone group. ${ }^{+}$Statistical differences $(P<0.1)$ between hydrocortisone group and control group. ${ }^{5}$ Statistical differences $(P<0.1)$ between dexamethasone group and control group. 
Table 2. Neurodevelopmental outcome at 2 years corrected age

\begin{tabular}{|c|c|c|c|c|c|c|c|c|c|c|}
\hline \multirow[b]{2}{*}{ Treatment group } & \multicolumn{3}{|c|}{ BSID-II MDI } & \multicolumn{3}{|c|}{ BSID-II PDI } & \multicolumn{3}{|c|}{ Touwen } & \multirow[b]{2}{*}{ Total } \\
\hline & Normal & Mildly delayed & Abnormal & Normal & Mildly delayed & Abnormal & Normal & Mildly abnormal & Abnormal & \\
\hline Hydrocortisone $(n=17)$ & 9 & 3 & 2 & 4 & 5 & 5 & - & - & - & 14 \\
\hline Controls $(n=22)$ & 13 & 3 & 0 & 10 & 3 & 3 & 3 & 0 & 0 & 19 \\
\hline
\end{tabular}

Data are summarized as frequencies (n). BSID-II, Bayley Scales of Infant Development, second edn.; MDI, Mental Developmental Index; PDI, Psychomotor Developmental Index. Based on BSID-II, children were classified as normal ( $\geq 85$ ), mildly delayed (70-84), or abnormal (<70). Based on Touwen, children were classified as normal, mildly abnormal (signs of minor neurological dysfunction), or abnormal (signs of cerebral palsy).

a

\begin{tabular}{|c|c|c|c|c|c|c|}
\hline infant ID & Day 0 & Day 1 & Day 2 & Day 7 & Term age & 3 Months PT \\
\hline 1 & & $x$ & & & & \\
\hline 2 & $X$ & $x$ & & & & \\
\hline 3 & $X$ & & & & 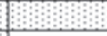 & \\
\hline 4 & & & & & $X$ & \\
\hline 5 & & & & & & \\
\hline 6 & & 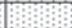 & & 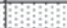 & 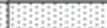 & $X$ \\
\hline 7 & & 4 & 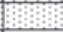 & & 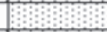 & $\mathrm{X}$ \\
\hline 8 & & 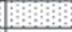 & $\mathrm{X}$ & & & \\
\hline 9 & & $x$ & $\theta$ & & & \\
\hline 10 & & $X$ & $X$ & & & \\
\hline 11 & & $X$ & $\mathrm{X}$ & & & \\
\hline 12 & & & & & & \\
\hline 13 & & & & $x$ & & \\
\hline 14 & & & $x$ & $X$ & & \\
\hline 15 & & 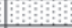 & 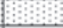 & 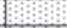 & & \\
\hline 16 & & & & & $X$ & \\
\hline 17 & It: & $X$ & & 12 & & $X$ \\
\hline
\end{tabular}

b

\begin{tabular}{|c|c|c|c|c|c|c|}
\hline infant ID & Day 0 & Day 1 & Day 2 & Day 7 & Term age & 3 Months PT \\
\hline \multicolumn{7}{|c|}{1} \\
\hline 2 & & $\mathrm{X}$ & & & & \\
\hline \multicolumn{7}{|c|}{3} \\
\hline \multicolumn{7}{|c|}{4} \\
\hline \multicolumn{7}{|c|}{5} \\
\hline \multicolumn{7}{|c|}{6} \\
\hline \multicolumn{7}{|c|}{7} \\
\hline \multicolumn{7}{|c|}{8} \\
\hline 9 & & & & & & $X$ \\
\hline 10 & & & & & & 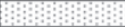 \\
\hline \multicolumn{7}{|l|}{11} \\
\hline \multicolumn{7}{|l|}{12} \\
\hline \multicolumn{7}{|c|}{13} \\
\hline \multicolumn{7}{|c|}{14} \\
\hline \multicolumn{7}{|c|}{15} \\
\hline 16 & & $X$ & & & & \\
\hline 17 & & & & & & \\
\hline
\end{tabular}

Figure 1. Individual trajectories of general movements (GMs) in the (a) hydrocortisone (HC) group and the (b) dexamethasone (DXM) group. Quality of GMs before HC or DXM treatment commenced (day 0), $1 \mathrm{~d}$ (day 1), $2 \mathrm{~d}$ (day 2), $1 \mathrm{wk}$ (day 7) after first dose, at term age, and around 3 months postterm (PT). Normal GMs or fidgety movements (FMs) (white); poor repertoire (dotted); chaotic (gray); cramped-synchronized (dark gray); hypokinetic (black). At 3 months PT: abnormal FMs (dotted); absent FMs (dark gray). Crosses represent missing recordings.

to be significantly lower than MOS on day 0 in the DXM group (median 10 vs. $11, P=0.030$ ) only on day 1 . The changes in MOS did not differ significantly between the two groups.

\section{Multivariate Analysis}

HC infants had a higher median MOS at 3 months after term than DXM infants. We assessed the relationship between MOS and treatment with either HC or DXM using multivariate linear regression allowing for possible confounders. First, we transformed the outcome variable MOS because the MOS was not normally distributed for the group as a whole (KolmogorovSmirnov test $P<0.001)$. By squaring the MOS, the new variable had a more normal distribution (Kolmogorov-Smirnov test $P=$ $0.153)$. Clinical variables, considered to be possible confounders with $P<0.1$, included birth weight, gestational age, Apgar score at $5 \mathrm{~min}$, and mean and cumulative equivalent doses of cortisol. After entering these variables as predictors and group assignment (as dummy variables) in the multivariate regression model, only gestational age and DXM treatment remained significant, with an explained variance of $20.2 \%$. We present the results in Table 3. After adjusting for the squared MOS, infants who received DXM had a reduction in MOS of well over two-thirds of a point (0.7) at 3 months of age as compared to infants who received HC.

\section{DISCUSSION}

In this longitudinal, observational study, we found a difference in GM quality in preterm infants who were postnatally treated with either HC or DXM. This difference was evident on the first day following treatment and at around 3 months after term. We found a higher MOS in the HC group, which reached statistical significance at 3 months after term. Furthermore, in the DXMtreated infants, MOS on the first day following treatment had decreased compared to their MOS before treatment. This was not the case for the HC-treated infants.

The first and most important finding of our study was that HC infants had a higher MOS at 3 months after term than DXM infants. Previously, Einspieler et al. reported that impairment of the quality of the motor repertoire, especially around 3 months after term, is highly predictive of severe neurological sequelae $(11,13)$. Of the five items on the Motor Optimality List, FM quality is the most reliable predictor of either severe neurological abnormalities or a normal neurological outcome $(13,16)$. In our study, however, we found no difference in the quality of FMs between the two groups. As a consequence, we need to turn to the remaining items of the Motor Optimality List to explain the difference in MOS. Previously, Bruggink et al. reported that MOS distinguished between preterm infants who showed a normal neurological outcome, infants who developed minor neurological dysfunction, and infants who developed cerebral palsy (15). This held true even if the quality of FMs was excluded from MOS. Thus, the difference we found in MOS may contribute to more neurological impairment at school age in children who had been treated with DXM in the neonatal period. 

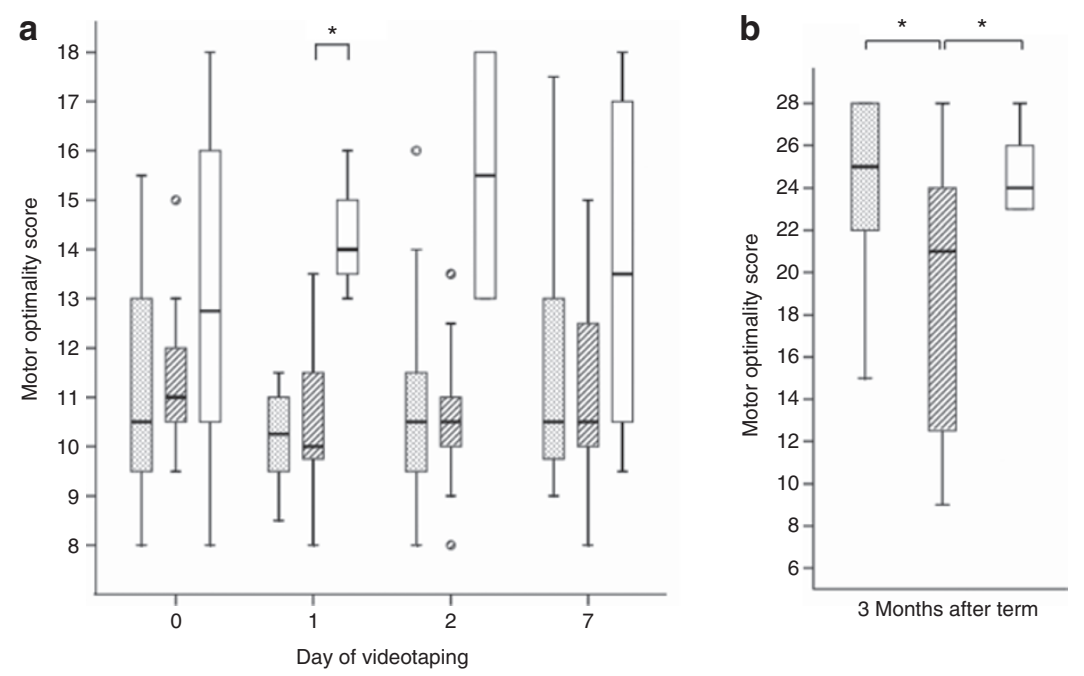

Figure 2. Motor optimality scores (unadjusted) (a) until the first week after treatment commenced and (b) at 3 months after term in hydrocortisone infants (dotted), dexamethasone infants (hatched), and controls (white). The data in the graphs are presented as box and whisker plots. Boxes represent the individual values between the 25 th and 75 th centiles (interquartile range); whiskers represent the range of the values, with the exception of outliers. Outliers are the circles, defined as values between 1.5 interquartile range and 3 interquartile ranges from the end of a box. On days 1 and 2, only 3 and 2 , respectively, recordings of controls were present. ${ }^{*} P<0.05$.

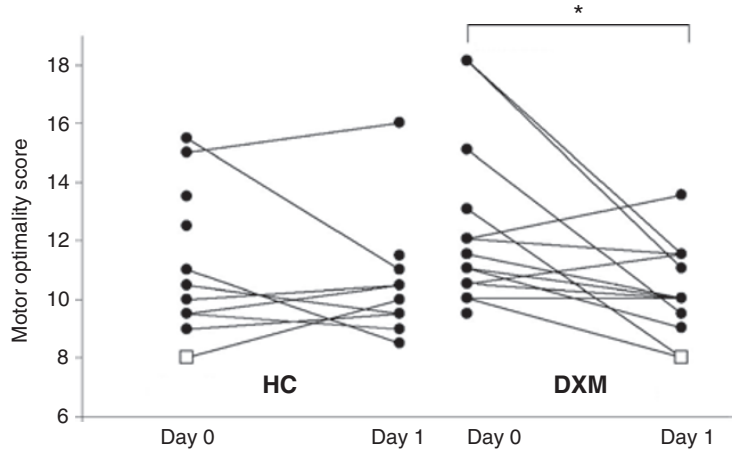

Figure 3. Motor optimality scores before therapy (day 0 ) and the first day following therapy (day 1 ) in both treatment groups. ${ }^{*} P<0.05$. Open box indicates motor optimality score in hypokinetic infant.

Table 3. Multiple regression of motor optimality score on birth weight and treatment group

\begin{tabular}{lccc}
\hline Variable & Coefficient $(\beta)$ & $t$ & $P$ value \\
\hline Constant & & -1.026 & 0.314 \\
Gestational age & 0.312 & 1.776 & 0.087 \\
Dexamethasone treatment & -0.515 & -2.935 & 0.007
\end{tabular}

Infants who received dexamethasone had a reduction in motor optimality score of 0.7 of a point as compared to infants receiving hydrocortisone. Per week increase in gestational age, the motor optimality score increased by 0.6 of a point.

Surprisingly, differences in MOS were not demonstrated before 3 months after term. A possible cause for this relatively late-occurring finding may be related to DXM having a low mineralocorticoid-mediated activity, high glucocorticoidmediated activity, and long biological half-life in comparison to HC (18). Animal studies provided evidence that the activation of glucocorticoid receptors induces damage to neurons, such as promoting apoptotic activity of neurons in the hippocampus. In contrast, mineralocorticoid receptors protect against apoptosis by upregulation of antiapoptotic proteins (19-22), suggesting that prolonged exposure to DXM treatment, but not HC, may alter hippocampal plasticity. We speculate that neuronal apoptosis does not immediately lead to such drastic changes in the neuronal architecture that GMs are affected in their quality. A more extended time course of neuronal apoptosis might be required before differences between HC and DXM come to light. Human studies that reported lower brain volumes at term-equivalent age after postnatal DXM therapy (23), and not after HC therapy (24), support this hypothesis.

In our study population, the HC infants had lower birth weights and gestational ages, higher Apgar scores, and received lower mean and cumulative equivalent cortisol doses. The higher Apgar scores and the lower cortisol doses could both have contributed to a higher MOS in the HC group. By performing multivariate linear regression, however, only gestational age and DXM treatment accounted for the difference in MOS. Besides, the HC infants had a higher MOS and they did not develop cerebral palsy at 2 years of age whereas some DXM infants did. Moreover, some brain ultrasound scans worsened following DXM treatment. Our results were in line with studies investigating long-term effects of postnatal DXM and HC therapy on neurological outcome at school age (3-8). They suggested an increased risk of motor disorders in DXM infants whereas $\mathrm{HC}$ seemed to have no adverse effects on long-term neurological outcome. A higher MOS in the HC infants possibly indicated that $\mathrm{HC}$ had less adverse effects on neurodevelopment at an early age than DXM.

The second important finding of our study was that a significant decrease of MOS occurred only on the first day following therapy in DXM-treated infants and not in HC-treated infants. This finding replicated other findings previously reported for DXM-treated infants (16). Our study 
indicated that $\mathrm{HC}$ had no such effects on the GMs, possibly due to the lower glucocorticoid activity. It is known that glucocorticoids inhibit glucose uptake into muscle and nerve cells within $24 \mathrm{~h}$ (25). Glucose transport is also inhibited in hippocampal neurons and glia cells (19). This inhibition is mediated by the glucocorticoid receptor, which can be blocked by a glucocorticoid receptor antagonist but not by a mineralocorticoid receptor antagonist. Thus, only the glucocorticoid receptor is involved in the process of glucose inhibition. As mentioned previously, the glucocorticoid activity of $\mathrm{HC}$ is relatively low compared to DXM. Considering the lower inhibition of glucose uptake as a result of lower glucocorticoid activity, we speculated that this might explain why MOS was not affected on the first day following treatment in the HC-group infants.

We recognize several limitations of our study. First, the number of infants in our study was relatively small and, as a consequence, the results should be interpreted with caution. Second, the two study groups were not randomly assigned to either University Medical Centre Utrecht or University Medical Center Groningen. Despite the fact that the indication for corticosteroid therapy was identical (ventilator dependency after the seventh day of life), type of treatment (HC vs. DXM) depended on the hospital where the infant was treated. Third, we pooled the controls at both study sites into one group. Both centers are located in the Netherlands and are equipped with level-III obstetrics departments and neonatal intensive care units. Of course, there are small local differences; however, both centers work according to national provided guidelines and protocols provided by the Dutch Pediatrics and Obstetrics Associations. So antenatal and neonatal treatment policies were reasonably uniform. Moreover, no significant clinical differences between the two groups were noticed. Hence, we assume that the clinical care provided was comparable between these two hospitals and that combining these data was justified. Fourth, infant recruitment took place over an extended period of time. We attempted to limit the confounding effect of improved care by at least ensuring that enrollment of controls occurred over the same time span as the enrollment of patients. Furthermore, this study had limited data on MRI findings because in the late 90s, MRI was not yet a part of standard clinical care. Therefore, we were not able to associate our findings on GMs with detailed neuroimaging as found previously $(23,24)$. Still, we do not expect to have missed major brain lesions that could have resulted in deterioration of GM quality, as cranial ultrasound is able to detect most abnormalities that are associated with abnormal neurodevelopmental outcome $(26,27)$.

Our findings might have implications for BPD treatment. They suggest that HC therapy has preference over DXM for treating preterm infants at risk for BPD. Nevertheless, randomized clinical trials are required to further substantiate our findings.

In conclusion, we found that the quality of the motor repertoire at 3 months after term of HC-treated preterm infants was more optimal than that of DXM-treated preterm infants. In addition, the MOS decreased considerably on the first day following therapy only in the DXM infants. It is possible our findings were due to glucocorticoid receptor-mediated neuronal toxicity triggered by the inhibition of glucose transport in neurons and glia cells, and by increased apoptosis. Although the differences were small, the results of our study suggest that in BPD treatment, HC may have preference over DXM.

\section{METHODS}

\section{Study Design}

We performed a longitudinal, observational study in two centers, comparing the effect of postnatal HC and DXM therapy on GM quality. Control infants matched for gestational age at birth were selected from both centers. The infants were videoed before and after corticosteroid therapy commenced. From the video recordings, we assessed the quality of GMs off-line.

\section{Study Population}

The study population consisted of 56 preterm infants (born $<32$ weeks' gestation) who had been admitted to the neonatal intensive care unit of either University Medical Centre Utrecht or University Medical Center Groningen between 1992 and 2006. Of them, 17 were treated with HC (University Medical Centre Utrecht: starting dose $5 \mathrm{mg} / \mathrm{kg} / \mathrm{d}$ ) and 17 with DXM (University Medical Center Groningen: starting dose $0.5 \mathrm{mg} /$ $\mathrm{kg} / \mathrm{d})$. The remainder $(n=22)$ acted as controls and did not receive corticosteroid treatment. They were videoed in the same postnatal week as the DXM and HC groups.

Treatment indication was ventilator dependency after the seventh day of life. Both centers started treatment according to their center-bound treatment protocol. Nevertheless, dosage and schedule could vary depending on the clinical response of the individual infant. As a consequence, duration of corticosteroid therapy ranged from 1 to 12 weeks (median 22 days in the DXM group and 24 days in the HC group). Equivalent doses of cortisol (HC) were calculated by multiplying the dose of DXM by 26.7 (the ratio HC:DXM being 20:0.75) (18). Infants were excluded if they met one of the following criteria: major congenital anomalies, chromosomal disorders, or death before term age.

\section{Data Collection}

Characteristics were collected from medical records based on the entire admission period until discharge from the neonatal intensive care unit: gender, birth weight, gestational age, Apgar score at $5 \mathrm{~min}$, seizures, sepsis, intracranial hemorrhages detected by ultrasound (classified according to Papile et al. (28)) and periventricular densities (classified according to De Vries et al. (29)), sedative or anticonvulsant drugs, use of antenatal corticosteroids by the mother, treatment duration, postnatal day of starting therapy, mean equivalent cortisol dose, cumulative equivalent cortisol dose, BPD, and the revised version of the Nursery Neurobiologic Risk Score (30). BPD was defined as treatment with supplemental oxygen at 36 weeks postmenstrual age. We performed follow-up examinations at 2 years of age, consisting of the Bayley Scales of Infant Development, second edition (BSID-II) (31), and a neurological examination. In DXM infants, in whom BSID-II was not part of standard care, we instead performed a detailed standardized neurological examination based on Touwen (32). Children were classified as normal if the Mental Developmental Index or Psychomotor Developmental Index was $\geq 85$, mildly delayed/abnormal if the Mental Developmental Index or Psychomotor Developmental Index was between 70 and 84 (or signs of minor neurological dysfunction were seen), or abnormal if the Mental Developmental Index or Psychomotor Developmental Index was $<70$ (or signs of cerebral palsy were seen).

\section{Video Recording}

The infants were videoed on six occasions: prior to the beginning of treatment (day 0 ), on days 1,2 , and 7 following treatment, on reaching term age, and at 3 months after term. The recordings made on day 0 served as individual references. Recordings were made with 
the infants lying in the incubator in supine position and wearing only a diaper. Most infants could move their limbs and trunk freely, although some had intravenous lines. To enable reliable assessment of the infants' GMs, each infant was recorded for 30 to $60 \mathrm{~min}$. In case no GMs occurred, the quality of GMs was given the score hypokinetic. The recordings around 3 months of age were made during a normal outpatient visit or at home and lasted $10 \mathrm{~min}$, which is sufficiently long for a reliable assessment of FMs (10). Although interference was avoided as much as possible, any necessary actions by caregivers were permitted. Recordings during which the infant was either fussing or crying, or sucking on a pacifier were discarded. A few recordings were missing due to logistic and/or patient-related problems ( $n=14$ in HC group, $n=3$ in DXM group). In addition, 5 recordings of $\mathrm{HC}$ infants could not be properly evaluated due to short recordings $(n=2)$, not lying in supine position $(n=1)$, or inappropriate state (sleepy $n=1$, crying $n=1$ ). Most of the controls were videoed weekly, which resulted in fewer recordings present on day $1(n=3)$ and day $2(n=2)$ as compared to day $0(n=22)$ and day $7(n=22)$.

\section{Analysis of GMs}

The GMs were assessed off-line by at least two observers in the order the recordings were made as described by Einspieler (10). At least one observer was unaware of the clinical course (M.M.H.). In addition, we determined the MOS using the GM Optimality List for Preterm GMs and Writhing Movements (33). MOS could range from 8 (low optimality) to 18 (high optimality). We used the Optimality List for Fidgety Movements (Assessment of Motor Repertoire - 2 to 5 months) (15) to analyze the quality of FMs and MOS at 3 months. During this period, MOS could range from 5 (low optimality) to 28 (high optimality). Previously, the interrater agreement was reported to be good; the kappa statistics vary from 0.89 to $0.91(14,15)$.

\section{Data Analysis}

We assessed the differences in the quality of GMs (normal vs. abnormal) between the HC and DXM infants and the controls on the six occasions with the Fisher exact test. We applied the Kruskal-Wallis test to compare MOS between the three groups on the six occasions. Where appropriate, we used the Mann-Whitney U test with Bonferroni adjustment for multiple testing, but not for our comparisons of the DXM and HC groups, because these were the two groups under study. We did this because strict application of the Bonferroni adjustment in this particular instance might have led to true differences being judged as statistically not significant.

To determine the change in MOS on the first day following treatment for each treatment group separately, we used the Wilcoxon signed-rank test. We used the Mann-Whitney $U$ test to test whether this change in MOS differed significantly between the two groups and we performed multivariate linear regression to adjust for confounding factors. The independent variables included clinical variables that differed between the treatment groups with $P<0.1$. A two-sided $P$ value of $<0.05$ was considered statistically significant. When we applied the Bonferroni adjustment (three groups), we considered a $P$ value of $<0.017$ statistically significant. We used the Statistical Package for the Social Sciences 16.0 software for Windows (SPSS, Chicago, IL) to perform the statistical analyses.

\section{Ethics}

The study was approved by the institutional review boards of University Medical Centre Utrecht and University Medical Center Groningen. We obtained parents' informed consent prior to making the recordings.

\section{ACKNOWLEDGMENTS}

We greatly acknowledge the help of Hans Burgerhof for his statistical advice, Dr Titia Brantsma-van Wulfften Palthe in Utrecht for correcting the
English manuscript, and Helen Torrance for collecting the data of the included patients.

\section{STATEMENT OF FINANCIAL SUPPORT}

This study was part of the research program of the postgraduate school for Behavioral and Cognitive Neurosciences, University of Groningen. M.M.H. and A.M.R. were financially supported by grants of the Junior Scientific Master Class of the University of Groningen.

\section{REFERENCES}

1. Smith VC, Zupancic JA, McCormick MC, et al. Trends in severe bronchopulmonary dysplasia rates between 1994 and 2002. J Pediatr 2005; 146:469-73.

2. Doyle LW, Ehrenkranz RA, Halliday HL. Dexamethasone treatment after the first week of life for bronchopulmonary dysplasia in preterm infants: a systematic review. Neonatology 2010;98:289-96.

3. Halliday HL, Ehrenkranz RA, Doyle LW. Early ( $<8$ days) postnatal corticosteroids for preventing chronic lung disease in preterm infants. Cochrane Database Syst Rev 2010:CD001146.

4. Shinwell ES, Karplus M, Reich D, et al. Early postnatal dexamethasone treatment and increased incidence of cerebral palsy. Arch Dis Child Fetal Neonatal Ed 2000;83:F177-81.

5. Doyle LW, Ehrenkranz RA, Halliday HL. Dexamethasone treatment in the first week of life for preventing bronchopulmonary dysplasia in preterm infants: a systematic review. Neonatology 2010;98:217-24.

6. van der Heide-Jalving M, Kamphuis PJ, van der Laan MJ, et al. Short- and long-term effects of neonatal glucocorticoid therapy: is hydrocortisone an alternative to dexamethasone? Acta Paediatr 2003;92:827-35.

7. Lodygensky GA, Rademaker K, Zimine S, et al. Structural and functional brain development after hydrocortisone treatment for neonatal chronic lung disease. Pediatrics 2005;116:1-7.

8. Karemaker R, Heijnen CJ, Veen S, et al. Differences in behavioral outcome and motor development at school age after neonatal treatment for chronic lung disease with dexamethasone versus hydrocortisone. Pediatr Res 2006;60: $745-50$.

9. Doyle LW, Ehrenkranz RA, Halliday HL. Postnatal hydrocortisone for preventing or treating bronchopulmonary dysplasia in preterm infants: a systematic review. Neonatology 2010;98:111-7.

10. Einspieler C, Prechtl HF, Ferrari F, Cioni G, Bos AF. The qualitative assessment of general movements in preterm, term and young infants-review of the methodology. Early Hum Dev 1997;50:47-60.

11. Einspieler C, Prechtl HF. Prechtl's assessment of general movements: a diagnostic tool for the functional assessment of the young nervous system. Ment Retard Dev Disabil Res Rev 2005;11:61-7.

12. Ferrari F, Cioni G, Prechtl HF. Qualitative changes of general movements in preterm infants with brain lesions. Early Hum Dev 1990;23: 193-231.

13. Prechtl HF, Einspieler C, Cioni G, Bos AF, Ferrari F, Sontheimer D. An early marker for neurological deficits after perinatal brain lesions. Lancet 1997;349:1361-3.

14. Bruggink JL, Einspieler C, Butcher PR, Van Braeckel KN, Prechtl HF, Bos AF. The quality of the early motor repertoire in preterm infants predicts minor neurologic dysfunction at school age. J Pediatr 2008;153:32-9.

15. Bruggink JL, Einspieler C, Butcher PR, Stremmelaar EF, Prechtl HF, Bos AF. Quantitative aspects of the early motor repertoire in preterm infants: do they predict minor neurological dysfunction at school age? Early Hum Dev 2009;85:25-36.

16. Bos AF, Martijn A, van Asperen RM, Hadders-Algra M, Okken A, Prechtl HF. Qualitative assessment of general movements in high-risk preterm infants with chronic lung disease requiring dexamethasone therapy. J Pediatr 1998;132:300-6.

17. Bos AF, Dibiasi J, Tiessen AH, Bergman KA. Treating preterm infants at risk for chronic lung disease with dexamethasone leads to an impaired quality of general movements. Biol Neonate 2002;82:155-8.

18. Ramrakha PS, Moore KP. Oxford Handbook of Acute Medicine, 2nd edn. New York: Oxford University Press, 2004:587.

19. Uno H, Eisele S, Sakai A, et al. Neurotoxicity of glucocorticoids in the primate brain. Horm Behav 1994;28:336-48. 
20. Almeida OF, Condé GL, Crochemore C, et al. Subtle shifts in the ratio between pro- and antiapoptotic molecules after activation of corticosteroid receptors decide neuronal fate. FASEB J 2000;14:779-90.

21. Sousa N, Cerqueira JJ, Almeida OF. Corticosteroid receptors and neuroplasticity. Brain Res Rev 2008;57:561-70.

22. Huang CC, Lin HR, Liang YC, Hsu KS. Effects of neonatal corticosteroid treatment on hippocampal synaptic function. Pediatr Res 2007; 62:267-70.

23. Murphy BP, Inder TE, Huppi PS, et al. Impaired cerebral cortical gray matter growth after treatment with dexamethasone for neonatal chronic lung disease. Pediatrics 2001;107:217-21.

24. Benders MJ, Groenendaal F, van Bel F, et al. Brain development of the preterm neonate after neonatal hydrocortisone treatment for chronic lung disease. Pediatr Res 2009;66:555-9.

25. Ng PC. The effectiveness and side effects of dexamethasone in preterm infants with bronchopulmonary dysplasia. Arch Dis Child 1993;68(3 Spec No):330-6.

26. Leijser LM, de Bruïne FT, Steggerda SJ, van der Grond J, Walther FJ, van Wezel-Meijler G. Brain imaging findings in very preterm infants throughout the neonatal period: part I. Incidences and evolution of lesions, comparison between ultrasound and MRI. Early Hum Dev 2009;85:101-9.

27. De Vries LS, Van Haastert IL, Rademaker KJ, Koopman C, Groenendaal F. Ultrasound abnormalities preceding cerebral palsy in high-risk preterm infants. J Pediatr 2004;144:815-20.

28. Papile LA, Burstein J, Burstein R, Koffler H. Incidence and evolution of subependymal and intraventricular hemorrhage: a study of infants with birth weights less than 1,500 gm. J Pediatr 1978;92:529-34.

29. de Vries LS, Eken P, Dubowitz LM. The spectrum of leukomalacia using cranial ultrasound. Behav Brain Res 1992;49:1-6.

30. Brazy JE, Eckerman CO, Oehler JM, Goldstein RF, O’Rand AM. Nursery Neurobiologic Risk Score: important factor in predicting outcome in very low birth weight infants. J Pediatr 1991;118:783-92.

31. BayleyN. Bayley Scales of Infant Development, 2nd edn. San Antonio: The Psychological Corporation, 1993.

32. Touwen BCL. Neurological Development in Infancy. Clinics in Developmental Medicine, no. 58. London: Mc Keith Press, 1976.

33. de Vries NK, Erwich JJ, Bos AF. General movements in the first fourteen days of life in extremely low birth weight (ELBW) infants. Early Hum Dev 2008;84:763-8. 